\title{
Transport and Deposition of Functionalized CdTe Nanoparticles in Saturated Porous Media
}

\author{
Saeed Torkzaban, Yongman Kim, Martin Mulvihill, Jiamin Wan, Tetsu K. Tokunaga \\ Earth Sciences Division, Lawrence Berkeley National Laboratory, Berkeley, CA, U.S.A.
}

\begin{abstract}
Comprehensive understanding of the transport and deposition of engineered nanoparticles (NPs) in subsurface is required to assess their potential negative impact on the environment. We studied the deposition behavior of functionalized quantum dot (QD) NPs (CdTe) in different types of sands (Accusand, ultrapure quartz, and iron-coated sand) at various solution ionic strengths (IS). The observed transport behavior in ultrapure quartz and iron-coated sand was consistent with conventional colloid deposition theories. However, our results from the Accusand column showed that deposition was minimal at the lowest IS (1 mM) and increased significantly as the IS increased. The effluent breakthrough occurred with a delay, followed by a rapid rise to the maximum normalized concentration of unity. Negligible deposition in the column packed with ultrapure quartz sand $(100 \mathrm{mM})$ and Accusand $(1 \mathrm{mM})$ rules out the effect of straining and suggests the importance of surface charge heterogeneity in QD deposition in Accusand at higher IS. Data analyses further show that only a small fraction of sand surface area contributed in QD deposition even at the highest IS (100 mM) tested. The observed delay in breakthrough curves of QDs was attributed to the fast diffusive mass transfer rate of QDs from bulk solution to the sand surface and QD mass transfer on the solid phase. Scanning electron microscopy (SEM) and energy-dispersive X-ray (EDX) analysis were used to examine the morphology and elemental composition of sand grains. It was observed that there were regions on the sand covered with layers of clay particles. EDX spectra collected from these regions revealed that $\mathrm{Si}$ and $\mathrm{Al}$ were the major elements suggesting that the clay particles were kaolinite. Additional batch experiments using gold NPs and SEM analysis were performed and it was observed that the gold NPs were only deposited on clay particles originally on the Accusand surface. After removing the clays from the sand surface, we observed negligible QD deposition even at $100 \mathrm{mM}$ IS. We proposed that nanoscale charge heterogeneities on clay particles on Accusand surface played a key role in QD deposition. It was shown that the value of solution IS determined the extent to which the local heterogeneities participated in particle deposition.
\end{abstract}

\section{Introduction}

Recent increase in production of engineered nanoparticles (NPs) has raised concerns about their release to the environment and the associated risks to human health and ecosystem (Maynard, 2006). Quantum dots (QDs) are one example of novel engineered NPs that may be used for applications such as solar energy conversion, medical diagnostics, drug delivery, and lightemitting diodes (Bruchez et al., 1998; Luque et al., 2007; Murray et al., 1993). QDs are semiconductor nanocrystals with diameters in the range of 1-10 nm that exhibit unique optical and electrical properties not present in their bulk counterparts (Alivisatos, 1996). QDs consist of a metalloid crystalline core (e.g., CdTe, CdSe) and often a protective shell (e.g., ZnS, CdS). 
The nanoparticles can then be made water soluble by encapsulation in hydrophilic polymers containing carboxylic acid groups (Zhivko et al., 2006).

QDs have been demonstrated to exhibit cytotoxic effects (Hirschey et al., 2006; Kloepfer et al., 2005); hence a comprehensive understanding of the fate and transport of these materials in subsurface environment is required to assess their potential negative risk. To date, a limited number of studies have been conducted to investigate the mobility and deposition of engineered NPs (e.g. carbon nanotubes, iron NPs, Fullerene) in the subsurface environment (Jaisi et al., 2008; Kanel et al., 2007; Lecoanet et al., 2004; Pelley and Tufenkji, 2008; Saleh et al., 2007). To the best of our knowledge, none have investigated transport of functionalized QDs in porous media. It has been observed that the deposition of other NPs, similar to microsize colloidal particles, is controlled by their physicochemical properties and environmental conditions such as flow velocity, grain size, solution chemistry (Lecoanet and Wiesner, 2004; Quevedo and Tufenkji, 2009; Wang et al., 2008a,b; Li et al., 2008). Hence, in aquatic environments with the prevalence of negatively charged substrates (Ryan and Elimelech, 1996), the deposition of negatively charged NPs such as functionalized QDs is expected to be inhibited by repulsive electrostatic interactions (unfavorable conditions for deposition). However, engineered NP deposition under unfavorable conditions have experimentally been observed to be many orders of magnitude greater than theoretical predictions based on the classical theory of Derjaguin-Landau-Verwey-Overbeek (DLVO theory) (Derjaguin and Landau, 1941; Verwey and Overbeek, 1948). Various attempts have been made to explain the anomalously high deposition rates observed under unfavorable conditions, including attachment in secondary and primary energy minima (Brant et al., 2006; Chen and Elimelech, 2006), surface charge heterogeneity ( $\mathrm{Li}$ et al., 2008), and staining (Jaisi et al., 2008). Of these explanations, surface charge heterogeneities seem to be the most plausible mechanism for the deposition of NPs on the net negatively charged mineral surfaces. Moreover, it was shown that minor degrees of charge heterogeneity of sand surface resulted in a high NP deposition and the deposition rate decreased substantially over the course of NP introduction to the porous media (Wang et al., 2008a).

The role of surface charge heterogeneity on mineral surfaces in colloid deposition under unfavorable chemical conditions is well known (Johnson and Elimelech, 1995; Ryan and Elimelech, 1996). Most mineral surfaces in aqueous media have various surface functional groups and impurities bound to the surface which results in charge heterogeneity (Sposito, 2008). These heterogeneous sites may vary in scale from molecular to macroscopic levels (Sposito, 2008). It has been shown that the deposition of colloidal particles is less sensitive to solution IS when the size of surface charge heterogeneities is larger than the colloidal particles (Song et al., 1994). However, if the favorable sites are much smaller than the depositing particles, it is likely that the repulsive forces from neighboring regions have significant effect on deposition. Indeed, Kozlova and Santore (2007) experimentally demonstrated that attachment of $0.5 \mu \mathrm{m}$ silica spheres to a net-negative and net-repulsive surface on which nanotextured positive patches $(10 \mathrm{~nm})$ were randomly distributed was sensitive to solution IS. In another study, it was shown that interaction of $25 \mathrm{~nm}$ latex particles with several mineral surfaces exhibited strong adsorption under unfavorable conditions in the presence of a trivalent Eu (III) (Filby et al., 2008). The authors attributed the particle deposition to local charge heterogeneities created by Eu (III) adsorption on the solid surface, which permitted cation bridging between negatively charged surfaces. Therefore it is expected that small scale charge heterogeneity (e.g. nanoscale) which is ubiquitous on most natural surfaces is a key factor controlling the deposition of engineered NPs in subsurface environments.

The objective of this study was to elucidate the significant role of charge heterogeneity on transport and deposition of functionalized QDs in porous media under unfavorable conditions. Column transport experiments with negatively charged functionalized QDs at various solution ionic strengths (IS) were conducted using different types of silica sands (Accusand, ultrapure quartz, and iron-coated Accusand). A mathematical model, which takes into account a kinetic rate expression incorporating a dynamic blocking function for QD deposition onto the sand surfaces, was used to study the transport and deposition mechanisms. Scanning electron microscopy (SEM) and energy-dispersive X-ray (EDX) analysis were used to examine the morphology and elemental composition of sand grains. Additional batch experiments using gold NPs were performed, and the distribution of attached gold NPs on the sand surface was studied by SEM in order to shed light on the importance of localized charge heterogeneity on particle deposition. We propose that charge heterogeneities (presumably in nanoscale size), mainly on clay particles originally deposited on the sand surface, play a key role in the determination of QD transport and deposition. Our results suggest that the value of solution IS determines the extent to which the local heterogeneities participate in NP deposition.

\section{Material and methods}

\subsection{Preparation and characterization of $Q D s$}

Carboxyl ( $-\mathrm{COOH})$ functionalized CdS-capped CdTe QDs with diameters between 1 and $10 \mathrm{~nm}$ (determined by the manufacturer using transmission electron microscopy) were purchased from Vive Nano Inc. A very low solubility of CdTe in water has been reported (Li et al., 2007). Carboxyl functionalized $\mathrm{Au}$ particles with a mean $50 \mathrm{~nm}$ diameter and a concentration of $\sim 0.01 \% \mathrm{HAuCl} 4$ were obtained from Sigma Aldrich. The QD stock suspension with a Cd concentration of $18 \mathrm{mM}$ (determined by ICP-MS) was diluted by a factor of 5000 , unless otherwise mentioned, resulting in a final NP concentration of about $2.88 \times 10^{10}$ particle per ml. This value was obtained based on the average particle size of $50 \mathrm{~nm}$ (determined by dynamic light scattering) and CdTe density of $5.85 \mathrm{~g} / \mathrm{cm}^{3}$. Analytical reagent-grade $\mathrm{NaCl}$ (Fisher) and deionized (DI) water were used to prepare electrolyte solutions of varying IS (1-100 mM) buffered at $\mathrm{pH} 7.8$ with $0.5 \mathrm{mM} \mathrm{NaHCO} 3$ (Fisher). Prepared QD suspensions were stored at room temperature $\left(23-25{ }^{\circ} \mathrm{C}\right)$ for $1 \mathrm{~h}$ followed by filtering through a $100 \mathrm{~nm}$ nylon membrane (Whatman) in order to eliminate any possible aggregates prior to each experiment. The hydrodynamic diameter of the QDs was 
assessed using dynamic light scattering (DLS) (Brookhaven Instruments Corp., Holtsville, NY). The electrophoretic mobility (EPM) of the NPs was measured using a ZetaPlus analyzer (Brookhaven Instruments Corp., Holtsville, NY) and converted to a Zeta potential using the Smoluchowski equation. For size and EPM measurements, the stock solution was diluted 500 times in the electrolyte solution with the IS ranging 1-100 mM. Each measurement was performed in duplicate with each replicate repeated 10 times. Transmission electron microscopy (TEM) was used to confirm the measured size of the particles. Samples were prepared by placing a drop of a QD suspension on a 200 mesh copper grid with a carbon backing, which was allowed to dry overnight prior to analysis. The average particle diameter of the particles was determined by counting more than 45 particles from seven TEM images. TEM measurements were performed on a $200 \mathrm{KeV}$ Hitachi TEM.

\subsection{Porous media}

We selected two types of silica sand as the porous media for transport experiments, Accusand from Unimin Corporation (Le Sueur, MN), and ultrapure quartz sand with an impurity content of less than 35 ppm (Charles B. Chrystal Co., Inc, NY). The physical and chemical characterization of Accusand is given elsewhere (Schroth et al., 1996). The sand was cleaned to remove impurities such as organic matter by soaking in $70 \% \mathrm{HNO}_{3}$ for $16 \mathrm{~h}$, rinsing with DI water several times until $\mathrm{pH}$ equilibrated, followed by five cycles in a sonication bath for $20 \mathrm{~min}$ each. To remove fine colloidal materials, the supernatant was decanted and the sand was washed with DI water two times before resonication. The average grain diameter of the silica sands was determined to be $270 \mu \mathrm{m}$, with the diameter ranging from 250 to $300 \mu \mathrm{m}$. For transport experiments under favorable conditions for attachment, the clean Accusand was coated with iron oxide (goethite). Goethite particles were synthesized following a method described by Scheidegger et al. (1993). As it will be discussed later, the SEM imaging of the Accusand showed that some regions of the sand surface contained an abundance of clay platelets indicating our cleaning treatment was not efficient to eliminate the clay particles. For a set of experiments, the following cleaning protocol was employed to remove clays from the sand to study the effect of clays on deposition of the NPs: the sand was immersed into $0.5 \mathrm{M} \mathrm{NaCl}$ solution for $1 \mathrm{~h}$ in a sonication bath then flushed with DI water. Finally, the sand was added to excess DI water and sonicated for $1 \mathrm{~h}$. These steps (i.e. soaking in $\mathrm{NaCl}$ solution, DI water flushing, and sonication) were repeated until the turbidity of the supernatant was negligible as verified by UV/ Vis. After this intensive cleaning treatment, the SEM images showed that the sand surfaces were almost completely devoid of clays verifying that the combination of cation exchange and IS reduction was efficient to remove the majority of the clay particles.

The zeta potentials for silica surfaces are reported to be negative ranging from -100 to $-40 \mathrm{mV}$ as ionic strength increases from 0 to $100 \mathrm{mM}$ (Berli et al., 2003). In contrast, goethite particles are positively charged for the selected solution chemistries (Stumm, 1992).
Scanning electron microscopy (SEM) was used to study the general morphology of sand grains. Samples were prepared by dispersing a few sand grains on a silicon substrate. The measurements were carried out with a Zeiss Gemini Ultra-55 Analytical Scanning Electron Microscope operating at 2-5 KeV. To determine the elemental composition of the sand surface, an energy-dispersive spectroscopy (EDS) detector from EDAX was used together with the SEM.

\subsection{Column studies}

QD transport and deposition in water-saturated columns containing Accusand, ultrapure quartz, or goethite-coated sand were examined through a series of column experiments. The transport column was made of an acrylic tube, $10 \mathrm{~cm}$ long with an inner diameter of $25 \mathrm{~mm}$. The column was wetpacked and the bulk density $\left(\rho_{b}\right)$ and volumetric water content $(\theta)$ were determined independently from physical measurement of the column to be about $1.7 \mathrm{~g} \mathrm{~cm}^{-3}$ and 0.41 , respectively. The hydrodynamic dispersivity was obtained from the nonreactive tracer $\left(\mathrm{NaNO}_{3}\right)$ experiment to be $0.1 \mathrm{~cm}$. For QD transport experiments, the packed column was first flushed with 5 pore volumes (PV) of DI water using a syringe pump, and then equilibrated with 5 PVs of $\mathrm{pH}-$ buffered $(\mathrm{pH} 7.8)$ solution at the IS of interest. After equilibrating the $\mathrm{pH}$ and IS of the column, various pulses of QD suspension were introduced into the column (see Table 1 for the pulse duration of each experiment), which was followed by the injection of three PVs of QD-free solution at the same flow rate and IS. In each transport experiment, introduction of QD suspension (pulse duration) was continued until the normalized concentration of QDs reached unity in order to calculate the amount of surface area that contributed in particle deposition at a given IS. The superficial flow rate was $0.35 \mathrm{~cm} \mathrm{~min}^{-1}$ corresponding to an average pore-water velocity of $0.85 \mathrm{~cm} \mathrm{~min}^{-1}$ and a column residence time of $11.8 \mathrm{~min}$. This rather high velocity yielding a short residence time was employed to minimize the effect of any possible NP aggregation in our results. Finally, for selected experiments, the column was flushed with DI water (without QDs) after the completion of the transport experiments to investigate the release of the deposited QDs. Column effluent was collected at a constant interval using a fraction collector and analyzed for QD content by a UV/Visible spectrometer at a wavelength of $330 \mathrm{~nm}$. In representative experiments, ICP-

Table 1

Experimental conditions of QD transport studies conducted at various solution ionic strengths in saturated columns packed with different types of porous media.

\begin{tabular}{lclc}
\hline EXP. & $\begin{array}{l}\text { Ionic strength } \\
(\mathrm{mM})\end{array}$ & $\begin{array}{l}\text { Grain size } \\
\mathrm{d}_{50}(\mu \mathrm{m})\end{array}$ & $\begin{array}{l}\text { Pulse duration } \\
(\mathrm{PV})\end{array}$ \\
\hline IS effect (Accusand) & 1 & 270 & 2.4 \\
& 10 & & 2.3 \\
& 20 & & 2.7 \\
& 30 & & 2.7 \\
& 50 & & 4.6 \\
Grain size effect & 100 & 270 & 6.8 \\
(Accusand) & 50 & 135 & 4.6 \\
Ultrapure quartz & 50 & 270 & 2.3 \\
Iron-coated sand & 1 & & 33.5 \\
\hline
\end{tabular}


MS (Perkin Elmer DRC II) was used to measure the Cd concentration of the samples in order to verify the UV/Vis results.

\subsection{Batch experiments}

A series of batch experiments employing gold NPs were performed to examine the distribution of attached gold NPs on the sand surface. Batch experiments were conducted by placing $10 \mathrm{~g}$ of sand and $10 \mathrm{ml}$ of gold NP suspension (diluted 100 times from the stock suspension) into polypropylene centrifuge tubes. Three different solution IS (1, 50, and $100 \mathrm{mM}$ ) corresponding to the chemical conditions of the column were used. The NP suspension and sand were allowed to equilibrate for $2 \mathrm{~h}$ by gently rotating the tubes end over end $(15 \mathrm{rpm})$ on a tube rotator. At the end, the supernatant was decanted and the sand was washed with gold NP-free solution with the same IS three times. Finally the sand was dried in an oven $\left(60{ }^{\circ} \mathrm{C}\right)$ and samples were prepared for SEM analysis. It should be noted that the properties of gold NPs (e.g. surface charge and size) were slightly different from those of QDs. However, this complementary experiment was just performed in order to observe the distribution of deposited gold NPs on the sand surface. As it will be mentioned later, we were not able to detect any deposited QDs on the sand surface, although the effluent breakthrough curves showed significant deposition.

\subsection{Mathematical modeling}

The transport of QDs through water-saturated porous media can be described using the one-dimensional form of the advection-dispersion equation (ADE) that accounts for particle deposition onto solid-water interface:

$\frac{\delta C}{\delta t}=\lambda v \frac{\delta^{2} C}{\delta z^{2}}-v \frac{\delta C}{\delta z}-r_{d e p}$

where $C$ is the number of QDs per unit volume of the aqueous phase, $\lambda$ is the dispersivity (L), $v$ is the average pore-water velocity $\left(\mathrm{LT}^{-1}\right)$, and $r_{\text {dep }}$ is the mass transfer rate of QDs in aqueous phase to/from the deposited phase $\left(\mathrm{NL}^{-3} \mathrm{~T}^{-1}\right)$ given by:

$\rho_{b} \frac{\partial S}{\partial t}=r_{d e p}=\theta K_{d e p} \Psi_{s} C-\rho_{b} K_{d e t} S$

where $\rho_{b}$ is the soil bulk density $\left(\mathrm{ML}^{-3}\right), \theta$ is the water content $(-), K_{d e p}$ is the QD deposition coefficient $\left(\mathrm{T}^{-1}\right), \psi_{\mathrm{s}}$ is a dimensionless deposition function for deposited QDs which is related to the maximum QD deposition capacity $S_{\max }\left(\mathrm{NM}^{-1}\right)$, $S$ is the concentration of deposited QDs in the column $\left(\mathrm{NM}^{-1}\right)$, and $K_{\text {det }}$ is the first order detachment rate coefficient $\left(\mathrm{T}^{-1}\right)$.

A simple form for $\psi_{\mathrm{s}}$ is used in the model to account for time-dependent deposition behavior in a manner similar to Langmuirian blocking (Adamczyk et al., 1994):

$\Psi_{s}=\left(1-\frac{S}{S_{\max }}\right)$

When the value of $S_{\max }$ is large then this term approaches a value of 1 and time-dependent deposition behavior becomes irrelevant. In case of negligible detachment rate,
Eq. (2) reduces to an irreversible deposition kinetic. A modified version of HYDRUS-1D (Simunek et al., 2005) was used to solve Eqs. (1)-(3).

\section{Results and discussion}

\subsection{Size and zeta potential of nanoparticles}

To investigate the influence of physicochemical properties of QDs on their deposition behavior, the QD zeta potential was evaluated as a function of solution IS. At $\mathrm{pH} \mathrm{7.8,} \mathrm{the} \mathrm{zeta}$ potentials of the QDs increased (became less negative) from -45 to $-30 \mathrm{mV}$ as the IS increased from 1 to $100 \mathrm{mM}$. This can be attributed to stronger charge screening and compressed double layer thickness at higher IS. This negative surface charge is attributed to the presence of carboxyl functional groups in the ligand coating of the QDs (Quevedo and Tufenkji, 2009). The zeta potentials of the functionalized gold NPs ranged from -35 to $-25 \mathrm{mV}$ as the IS increased from 1 to $100 \mathrm{mM}$.

The mean diameters and particle size distributions of the QDs were studied over the range of IS (1-100 mM) using DLS. The DLS measurements (intensity-based average diameter) showed a mean diameter larger than $100 \mathrm{~nm}$ for QDs in all IS. However, when the NP suspension was filtered through a $100 \mathrm{~nm}$ filter, the difference in cadmium concentration measured by ICP-MS was negligible for filtered and unfiltered suspensions. When the QD suspensions were filtered through a $20 \mathrm{~nm}$ filter, the ICP-MS results showed that about $70 \%$ of NPs were filtered out. These findings show that the majority of QDs were smaller than $100 \mathrm{~nm}$ and larger than $20 \mathrm{~nm}$. It is worth mentioning that intensity-based size distributions do bias toward large particles as the light scattering of the particles is roughly proportional to the sixth power of particle diameter (Rayleigh approximation). After filtering the QD suspension through the $100 \mathrm{~nm}$ filter, the DLS measurements showed that the average diameters of QDs in all solution IS were around $50 \mathrm{~nm}$. Moreover, the NP suspensions were stable over the course of experiments verified by DLS and UV/Vis measurement. These results are in agreement with the reported diameters of QDs and their stabilities in the literature (Quevedo and Tufenkji, 2009). The TEM micrographs (Fig. 1) showed equi-axed QD particles with an average diameter of $8 \mathrm{~nm}$. The

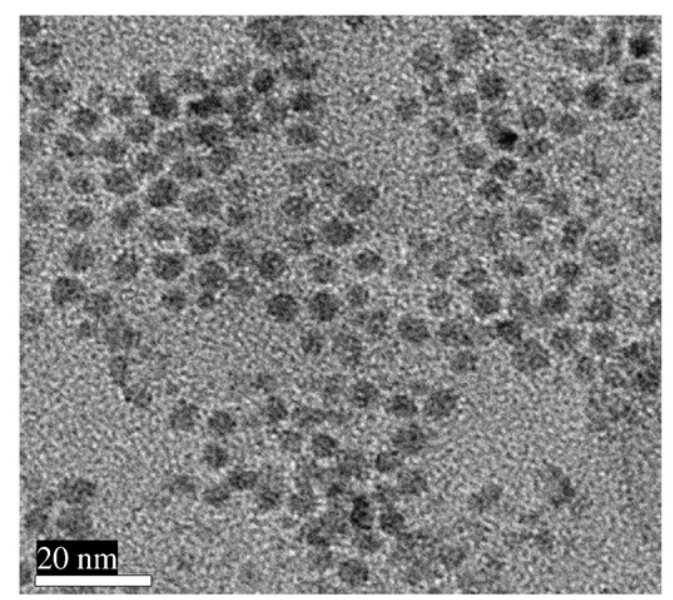

Fig. 1. TEM image of QDs. 


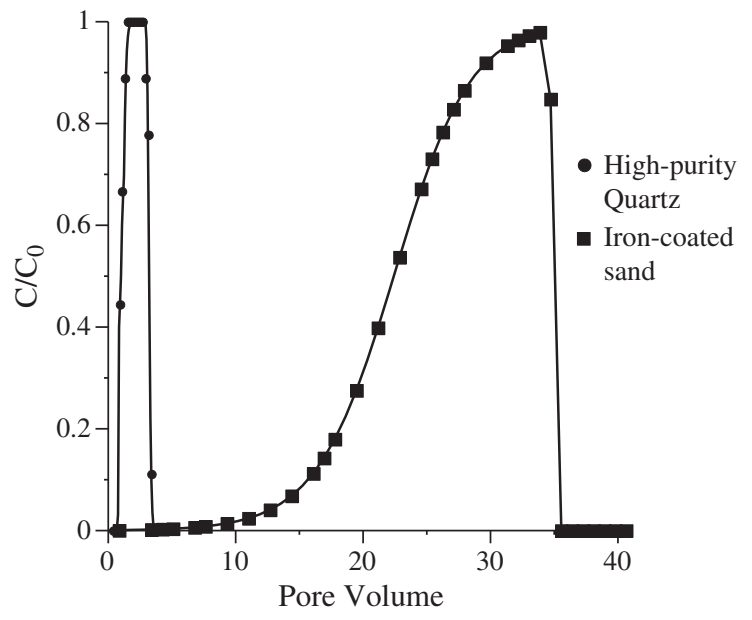

Fig. 2. Measured and fitted breakthrough curves of representative QD transport experiments in saturated columns packed with ultrapure quartz and iron-coated sand. Experimental conditions are: solution ionic strength for high-purity quartz $=100 \mathrm{mM}$ and for iron-coated $=1 \mathrm{mM}$, average pure water velocity $=12.5 \mathrm{~m} /$ day, column length $=10 \mathrm{~cm}$, and porosity $=0.41$, $\mathrm{pH}=7.8$. Other experimental parameters relevant to the column experiments are given in Table 1.

comparison of TEM and DLS data indicates that a few NPs probably formed aggregation and also it should be noticed that the hydrodynamic diameters of the NPs are larger than the sizes determined by TEM due to the thickness of polymer coating and the surrounding electrical double layer.

\subsection{Deposition of $Q D s$ in sand columns}

Figs. 2-4 present representative measured and fitted breakthrough curves (BTCs) of QDs obtained from columns packed with different sands at various solution IS. In these figures the normalized effluent concentrations are plotted versus the number of pore volumes passed through the column. QD injection to the column in each experiment continued such that the effluent concentration reached the feed concentration. Table 1 summarizes experimental conditions and the number of pore volumes (pulse duration) at which the influent was switched to a QD-free solution.

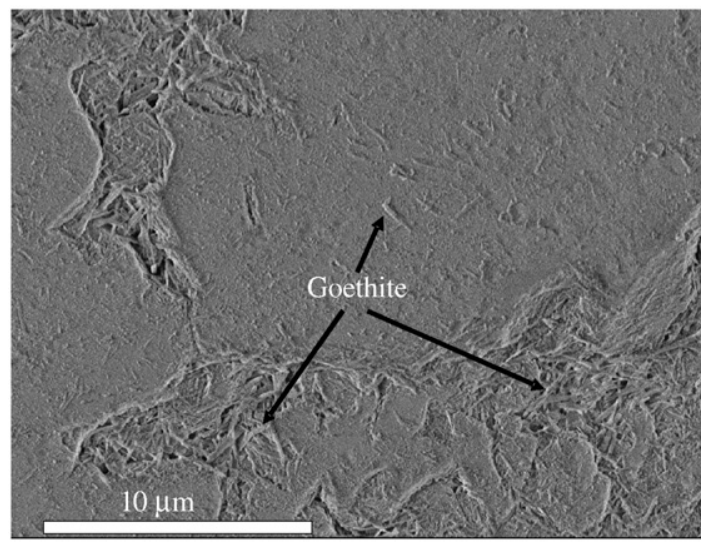

Fig. 3. SEM image of the Accusand surface coated with goethite particles. (a)

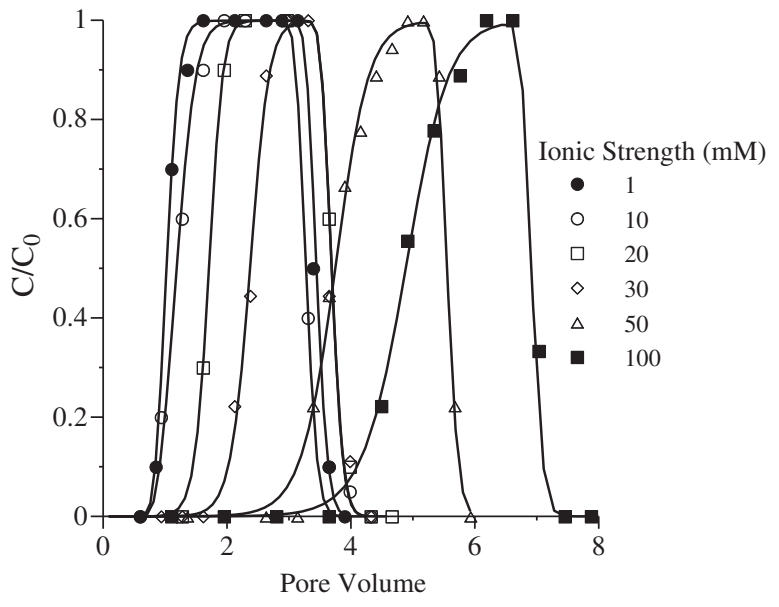

(b)

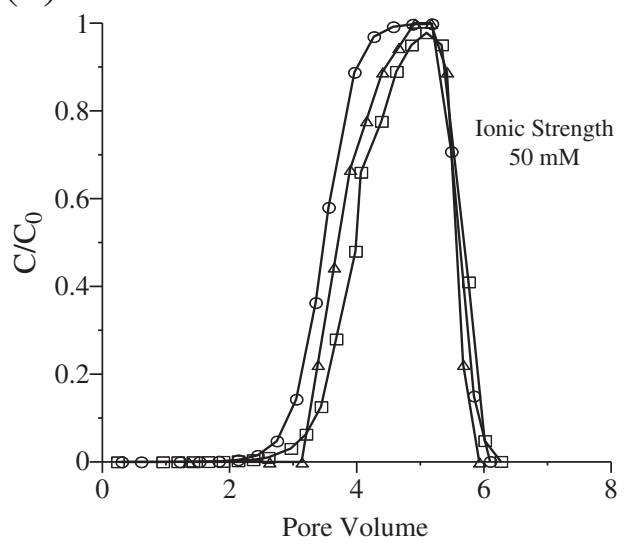

(c)

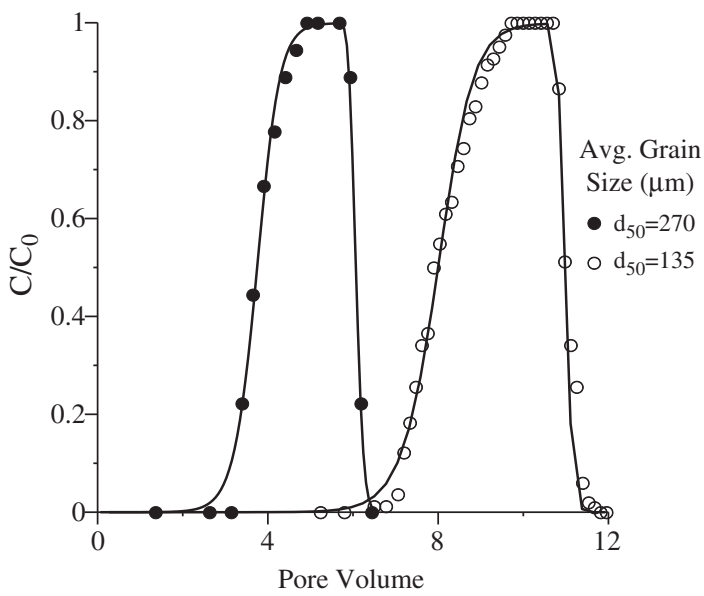

Fig. 4. Breakthrough curves of QD transport experiments conducted in columns packed with Accusand (a) representative measured and fitted breakthrough curves at various solution ionic strengths (b) triplicate BTCs obtained at ionic strength of $50 \mathrm{mM}$ are plotted together (c) measured and fitted breakthrough curves of two different grain sizes at ionic strength of $50 \mathrm{mM}$. Experimental conditions are: average pure water velocity $=12.5 \mathrm{~m} / \mathrm{day}$, column length $=10 \mathrm{~cm}$, and porosity $=0.41, \mathrm{pH}=7.8$. Other experimental parameters relevant to the column experiments are given in Tables 1 and 2. 
It is evident that QD deposition was negligible in column packed with ultrapure quartz sand conducted at solution IS of $100 \mathrm{mM}$ (Fig. 2). This result is in qualitative agreement with DLVO theory predicting no deposition in primary and secondary minima due to the negative values of zeta potentials of QDs and quartz sand in these chemical conditions. It is also concluded that straining, trapping of the NPs in small pores, did not occur in the silica sands employed in this study as the range of grain sizes is the same in both ultrapure quartz and Accusand. The QD effluent concentration obtained from a column packed with goethitecoated sand (microscale heterogeneity favorable for deposition) conducted at the IS of $1 \mathrm{mM}$ showed a significant deposition. In fact, the QD breakthrough occurred after injecting ca. 10 PVs of suspension, and approximately 34 PVs was required to attain the normalized concentration of 0.98 (Fig. 2). Note that the surface of goethite-coated sand was partially coated with colloidal-size goethite particles verified by SEM analysis (Fig. 3). Under the chemical conditions of the experiment presented here (i.e., $\mathrm{pH} 7.8$ ), the iron oxide patches (in microscale sizes) on the sand surface are positively charged, and thus provided favorable surfaces for the deposition of the negatively charged QDs.

It is observed that QD deposition was very little at the lowest IS ( $1 \mathrm{mM})$, but it increased significantly as the IS increased for Accusand columns (Fig. 4a). Reproducibility of the transport experiments is shown by plotting triplicate BTCs of QDs obtained at IS of $50 \mathrm{mM}$ (Fig. 4b). Note that the BTCs are atypical and did not occur after 1 pore volume, as commonly observed in studies with colloids. The delay in the breakthrough, which is more pronounced at higher IS, followed by a relatively rapid rise to the normalized concentration of one. This suggests the occurrence of either NP detachment from the sand surface or filling (blocking) of all available sites for deposition. Blocking of available sites for deposition seems to be a more likely explanation, because detachment produced insignificant tailing in the breakthrough curves.

An additional experiment was conducted to better understand the importance of the extent of surface area on QD deposition. Fig. 4c presents the BTCs obtained from column packed with Accusand of different sizes (135 and $275 \mathrm{~mm}$ ) at IS of $50 \mathrm{mM}$ along with model simulations. The result shows that the BTC obtained from fine sand exhibits the same behavior as that of coarse sand. However, the QD deposition increased about two times in fine sand compared with coarse sand as the BTC occurred at a later time (6 PVs for the fine sand versus 3 PVs for the coarse one). This confirms that when the specific surface area of the sand increases roughly about two times, the deposition also increases by a factor of two, implying the importance of the amount of favorable sites on the sand surface. It should be noted that NP straining (i.e. small pores, and regions close to stagnation points) and deposition in dead-end pores were not important in our study because a negligible deposition was observed in ultrapure quartz sand at IS of $100 \mathrm{mM}$ and also in Accusand in IS of $1 \mathrm{mM}$.

To quantitatively compare the experiments, the QD breakthrough data were simulated with the solutions of Eqs. (1)-(3) and the fitted values of the initial deposition rate coefficient $\left(K_{d e p}\right)$ and maximum sorption capacity $\left(S_{\max }\right)$ are presented in Table 2 . The very good quality $\left(R^{2}\right.$ values exceeding $95 \%$ for most cases) of the model fit is illustrated in Figs. 2 and 4a,c presenting comparisons of measured (symbols) and fitted (solid lines) QD effluent concentrations for the representative column experiments. The model simulations confirm that assuming a Langmuir blocking model (Eq. (3)) was sufficient to closely fit the behavior of BTCs. QD attachment to the solid surface was governed by the blocking effect and that a Langmuir-type reaction closely modeled the behavior of BTCs. The fitted values of $K_{d e p}$ and $S_{\max }$ were found to be unique as the final values of fitting processes were not affected by initial values of the parameters. It is observed that the values of $K_{d e p}$ and $S_{\max }$ increased with IS (Table 2), reflecting the observed delay in breakthrough as the IS increases. $K_{\text {det }}$ was set to zero in the simulations because negligible tailing was observed in effluent concentration upon switching to QD-free solution.

The values of $S_{\max }$ obtained from simulations range from 0.01 to 0.94 ( $\mu$ g-CdTe/g-sand) as the IS increases from 1 to $100 \mathrm{mM}$. Using these values and the theoretical specific surface area of Accusand $\left(8.1 \times 10^{-3} \mathrm{~m}^{2} / \mathrm{g}\right)$, the fraction of the solid surface that contributed to QD deposition $\left(S_{f}\right)$ at a given IS was approximated and listed in Table 2 . The $S_{f}$ can also be estimated from the effluent BTC based on the following mass balance equation (Ko and Elimelech, 2000):

$S_{f}=\frac{\pi r_{p}^{2} r_{c} q\left(C_{0} T_{\text {pulse }}-\int_{0}^{T_{\text {total }}} C d_{t}\right)}{3(1-\theta)}$

where $r_{p}$ is the radius of the QD, $r_{c}$ is the radius of the sand, $q$ is the Darcy velocity, $C_{0}$ is the influent $\mathrm{QD}$ concentration, $T_{p u l s e}$ is the injection time, and $T_{\text {total }}$ is the entire time of the experiment. It is observed that the values of $S_{f}$ determined from the two approaches are very similar and small (Table 2). The results suggest that even at the highest IS tested only a minor fraction of sand surfaces was favorable for deposition.

The observed delays in the breakthrough at higher solution IS are due to high initial rates of particle deposition $\left(K_{d e p}\right)$. The high initial rates may be explained by the following two mechanisms: (1) a high diffusive mass transfer rate of QDs from bulk solution to the sand surface and (2) QD mass transfer on the unfavorable regions of the solid surface. The diffusive mass transfer rate of QDs, which is related to

Table 2

Simulation parameters obtained by fitting QD breakthrough curves of column experiments to the solution of Eqs. (1)-(3).

\begin{tabular}{|c|c|c|c|c|c|}
\hline EXP. & $\begin{array}{l}\text { IS } \\
(\mathrm{mM})\end{array}$ & $\begin{array}{l}S_{\max }^{\mathrm{a}} \\
(\mu \mathrm{g} / \mathrm{g}-\mathrm{sand})\end{array}$ & $\begin{array}{l}K_{d e p} \\
\left(\min ^{-1}\right)\end{array}$ & $\begin{array}{l}S_{f}^{\mathrm{b}} \\
(\mathrm{BTC}) \%\end{array}$ & $\begin{array}{l}S_{f}^{c} \\
\text { (fitted) \% }\end{array}$ \\
\hline IS effect & 1 & 0.01 & 0.03 & $<0.01$ & $<0.01$ \\
\hline \multirow[t]{5}{*}{ (Accusand) } & 10 & 0.05 & 0.13 & 0.01 & 0.01 \\
\hline & 20 & 0.17 & 0.65 & 0.05 & 0.04 \\
\hline & 30 & 0.33 & 0.86 & 0.1 & 0.08 \\
\hline & 50 & 0.66 & 0.94 & 0.19 & 0.17 \\
\hline & 100 & 0.94 & 1.03 & 0.31 & 0.23 \\
\hline $\begin{array}{l}\text { Grain size } d_{50} \\
\qquad(135 \mu \mathrm{m}) \\
\text { (Accusand) }\end{array}$ & 50 & 1.69 & 1.56 & 0.46 & 0.42 \\
\hline Ultrapure quartz & 50 & $\mathrm{ND}^{\mathrm{d}}$ & ND & ND & ND \\
\hline Iron-coated sand & 1 & 620 & 0.62 & 8.3 & 7.8 \\
\hline
\end{tabular}

${ }^{a}$ Maximum deposition capacity. ${ }^{\mathrm{b}, \mathrm{c}}$ Fraction of the solid surface that contributed to deposition. ${ }^{\mathrm{d}}$ Not determined. 
collector efficiency $(\eta)$ in filtration theory (Yao et al., 1971), can be quantified using correlation equations such as those of Tufenkji and Elimelech (2004). The value of $\eta$ for our system was determined to be 0.13 suggesting a very high diffusive mass transfer from bulk solution toward the solid surface. However, the high value of diffusive mass transfer rate cannot alone explain the high initial deposition rates because a minor fraction of surface area $\left(S_{f}\right)$ was available for deposition (see Table 2). Note that the diffusive mass transfer rate of particles multiplied by the favorable surface area $\left(S_{f}\right)$ results in deposition rates assuming no deposition on unfavorable regions of the sand. The very small values of favorable surface area $\left(S_{f}\right)$, even at $100 \mathrm{mM}$ IS, would indicate that unfavorable regions of the sand did not contribute in deposition. Therefore, we have proposed an additional mechanism, that is, QD mass transfer on the unfavorable regions of the solid surface. QDs that collide with the unfavorable regions of solid surface via diffusion and become weakly associated with the surface can be translated on the surface by hydrodynamic forces to find the favorable sites for deposition. Indeed, experimental evidence by Kuznar and Elimelech (2007) demonstrates that weakly interacting colloidal particles can be translated along the collector surface via hydrodynamic forces and be retained in regions near the rear stagnation point. The amount of colloid mass transfer on the solid surface is expected to increase with increasing IS (Torkzaban et al., 2008a, 2010). Such a retardation-type behavior of engineered NPs has also been reported (Wang et al., 2008a; Li et al., 2008). The shape of obtained BTCs in this study is in contrast with results from transport experiments on micrometer-size colloids in the literature (e.g. Bradford et al., 2005; Torkzaban et al., 2008b) which show that colloid BTCs generally occur after introducing one pore volume of colloid suspension. This may be explained by the small values of diffusive mass transfer of the microsize colloids.

\subsection{Surface charge heterogeneity}

It has been reported that surface charge heterogeneities on collector surfaces can control colloidal particle (e.g. latex particle, bacteria, NPs) deposition behavior under conditions deemed unfavorable for deposition (Johnson et al., 1996; Li et al., 2008). Surface charge heterogeneities on mineral surfaces may vary in scale from molecular to macroscopic levels. Comparison of the results obtained from ultrapure quartz sand with Accusand reveals the importance of surface charge heterogeneities on the surface of Accusand grains in QD deposition. In the case of quartz sand with unfavorable and homogenous surfaces, QD deposition was negligible even at $100 \mathrm{mM}$ solution IS. It is evident that the amount of the heterogeneities on the Accusand and their contribution was minor in QD deposition at the low solution IS. However, more heterogeneity was produced and began participating in QD deposition by increasing the solution IS. We propose the following hypothesis to explain the observed pattern. There were minor favorable sites distributed on the sand surfaces such that the sum of attractive van der Waals interaction and weak repulsive electrostatic interaction results in net
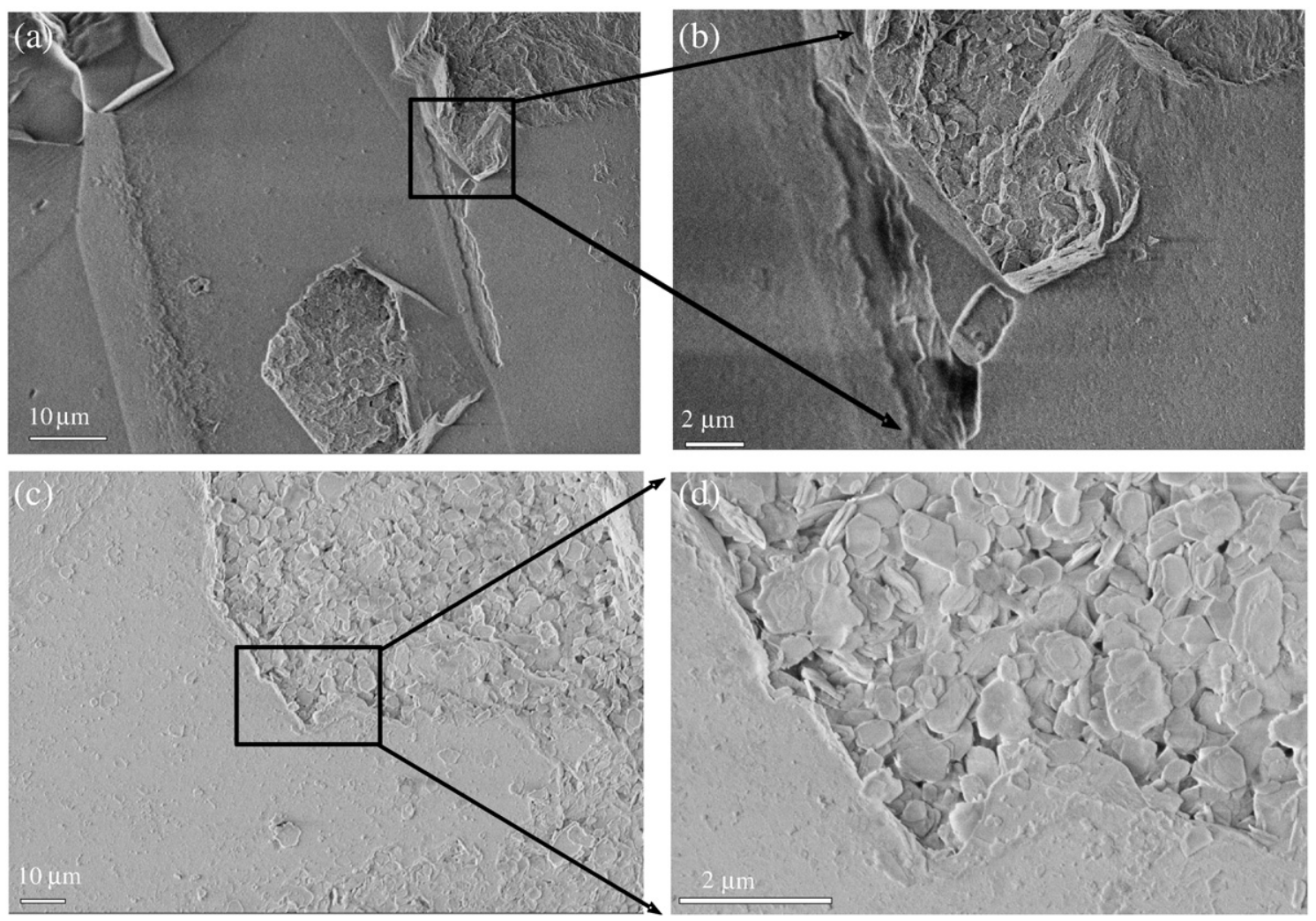

Fig. 5. SEM images of Accusand surfaces showing the abundance of kaolinite clay particles deposited heterogeneously on the surfaces of the sand grains (a-d). 
attractive interaction energy at a given solution IS. The double layer thickness is thick at low solution IS because of highly negative zeta potential of the dominant unfavorable regions surrounding a few favorable sites. Therefore, it is unlikely that the NPs approach the favorable sites where they can experience the attractive forces. However, as the solution IS increases, the double layer thickness decreases, and some of the NPs may approach the favorable sites and succeed in attachment.

In order to further study the underlying mechanism that controlled QD deposition, the sand surfaces were analyzed by SEM/EDX. Typical SEM images for Accusand are shown in Fig. 5 showing that some regions on the sand were covered with layers of clay particles. EDX spectra collected from these regions revealed that $\mathrm{Si}$ and $\mathrm{Al}$ were the major elements suggesting that the clay particles were kaolinite. The clay particles were only observed on the surface of the Accusand, while the ultrapure quartz sand appeared free from clays (not shown). To observe the distribution of deposited NPs on the surface of the Accusand with SEM, batch experiments employing QDs and gold NPs were conducted as described earlier. A few sand grains were randomly taken from the batch tubes at various IS, and their surfaces were carefully investigated with SEM. We were not able to find any attached QDs on the surface of clay or other regions of the sand, which is probably due to the very small crystalline core size of these particles (1-10 nm). However, the gold NPs (core size around $50 \mathrm{~nm}$ ) were easily spotted on the clay surfaces of sand grains (Fig. 6) equilibrated with the NP suspensions at $50 \mathrm{mM}$ IS. We found the deposited gold NPs only on the clay surfaces and did not find them on the smooth surface of sand grains which did not contain clay. The attached gold NPs were randomly and sparsely deposited on the clay surfaces, and only single particle was observed on a particular location. These observations may suggest that there were some nanoscale favorable sites on the surface of clay particles contributing in deposition. It is worthwhile to mention that we employed gold NPs in order to observe the distribution of deposited NPs on the surface of clay particles. We acknowledge that the surface properties of gold NPs are different from those of QDs, but they nevertheless provide support that some charge

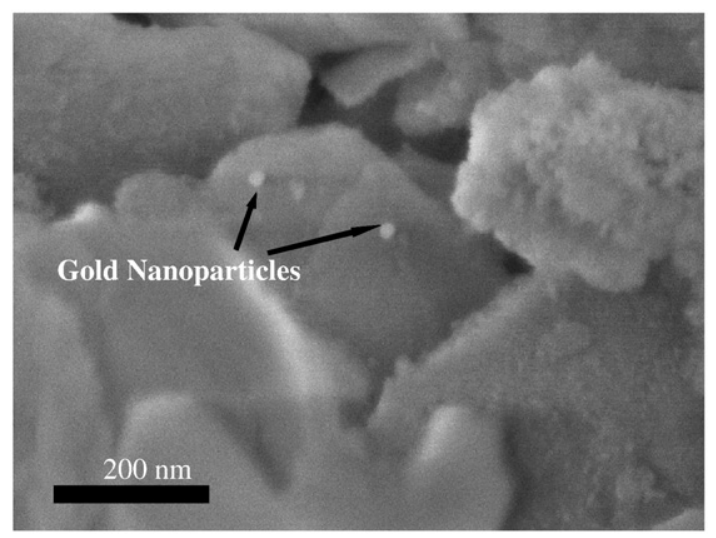

Fig. 6. High magnification SEM image showing attached gold nanoparticles on the surface of clays. heterogeneities on the surface of clay particles mainly contributed on NP deposition sites.

Indeed, when we flushed DI water through selected columns after completion of the transport experiment at a high solution IS, the effluent showed very high turbidity. To eliminate the clay particles released from the sand, a portion of the collected effluent samples was filtered through a $100 \mathrm{~nm}$ nylon membrane and then the filtrates were analyzed by ICP-MS for cadmium concentration. The unfiltered suspensions were also analyzed by ICP-MS after being digested with $70 \%$ nitric acid. The results showed a negligible concentration of cadmium in the filtered liquid, while a very high concentration of cadmium in the unfiltered suspensions was measured. This finding suggests that the deposited QDs in the column were mainly associated with clay particles on the sand grains. To further support our hypothesis that the QDs were mainly deposited on clay particles, an addition column experiment was conducted at $100 \mathrm{mM}$ IS using the extensively treated Accusand. As it was mentioned earlier, the Accusand was repeatedly soaked in $0.5 \mathrm{M} \mathrm{NaCl}$ solution followed by sonication in DI water until the turbidity of the supernatant became almost zero. The treated Accusand grains were analyzed with SEM, and the images showed that the majority of the clay particles were removed from the sand surfaces. The column experiments with the treated Accusand showed negligible QD deposition and the BTC occurred after one pore volume (data not shown). This finding clarifies the role of clay particles in QD deposition.

We believe that the favorable sites on clay particles of Accusand were in the range of nanoscale, because the SEM images of deposited gold NPs showed that the deposited gold NPs were randomly and sparsely distributed on the clay surfaces, and only single particle was observed on a particular location. Moreover, our results show that there were only minor amounts of favorable sites $\left(S_{f}\right)$ contributing in deposition even at the $100 \mathrm{mM}$ IS, less than $0.4 \%$, (Table 2). It is worth noting that these heterogeneities would not have a significant effect on the average zeta potential of the sand because the amounts of favorable sites were minor. If the surface charge heterogeneities on the clay particles were in microscale, the QD deposition on these sites would not be strongly dependent on IS which is in contrast with our result.

The repulsive forces from neighboring unfavorable regions of nanoscale charge heterogeneities have a significant effect on the adhesive forces exerted from nanoscale charge sites on the NPs (Duffadar and Davis, 2008; Duffadar et al., 2009). These researchers demonstrated through experimental and theoretical studies that nanoscale positively charged heterogeneities on negative surfaces produce colloid attachment depending on the solution IS and the size of colloidal particles (Duffadar and Davis, 2008; Duffadar et al., 2009). Kozlova and Santore (2007) experimentally demonstrated that $0.5 \mu \mathrm{m}$ silica particles attached to a net-negative and netrepulsive surface on which nanotextured positive patches $(11 \mathrm{~nm})$ were randomly distributed. Duffadar et al. (2009) through laboratory experiments and simulations showed that the interaction of flowing negative colloidal particles (0.5$2 \mu \mathrm{m}$ ) with polycation-based positive patches on negative substances caused particle deposition at high IS, however, no deposition occurred at low IS. Results presented by Duffadar and Davis (2008) indicate that the magnitude of the attractive 
electrostatic force between the colloids and the "favorable nanoscale patches" will increase with IS due to compression of the double layer thickness and will also be a function of the colloid size. Alonso et al. (2004) studied the interaction of negatively charged gold particles $(47 \mathrm{~nm})$ with granite mineral using the $\mu$ PIXE technique and demonstrated that NP attachment did not occur when there were no chemical heterogeneities on the solid surface. However, an attractive interaction between gold particles and positive Fe-minerals $(\sim 60 \mathrm{~nm})$ caused the particles to deposit on the net-repulsive granite surface.

\section{Conclusion and environmental implications}

Transport and deposition of engineered NPs in the natural environment determine the fate, bioavailability, and reactivity of these materials. This study provides insights into the deposition behavior of functionalized QDs under unfavorable attachment conditions, and identifies possible mechanisms controlling their deposition and release during flow through porous media. Our results suggest that charge heterogeneity, which is ubiquitous in most natural surfaces, and chemical conditions are key factors controlling the deposition rate of NPs onto mineral surfaces in subsurface environment. QD deposition onto favorable sites resulted in deposition rates much higher than those that would be predicted based on the average collector surface potential. The solution IS determines the extent of the mass transfer of QDs on the solid surface and the amount of deposition on the favorable sites.

The classical DLVO theory assumes that colloids and solid surfaces are perfectly smooth and homogeneous at the molecular level. In real systems, however, surface heterogeneities always exist and assumption of ideal homogeneous surfaces breaks down. Consideration of surface heterogeneity is of particular importance for NP deposition in the subsurface aquatic environment because of inherent charge heterogeneities at the molecular up to macro scales. Therefore, the interaction energies can no longer be described by a single value of interaction energy; rather, a distribution of interaction energies should be considered. Hence, proper characterization of surface heterogeneities of mineral surfaces may lead to improvements in our ability to predict deposition rates based on solution and surface chemistry.

On the basis of our findings, there is a potential for release of the deposited NPs due to detachment of clay particles if the solution IS decreases. In the natural subsurface environment, changes in solution chemistry can cause the detachment of clay particles from soil surfaces. For instance, a rainfall can result in a significant decrease in water IS, and consequently release of clay particles from the soil grain surfaces. This process may potentially increase the risk of groundwater contamination by ENPs, although the magnitude of such risk remains to be determined.

\section{Acknowledgments}

Funding was provided through the joint BER-EPA-NSF Nanoparticulate Research Program of the Office of Biological and Environmental Research, U.S. Department of Energy, under contract DE-AC02-05CH11231. The authors are grateful to three anonymous referees for their critical reviews and valuable comments that led to the improvement of the manuscript.

\section{References}

Adamczyk, Z., Siwek, B., Zembala, M., Belouschek, P., 1994. Kinetics of localized adsorption of colloid particles. Adv. Colloid Interface Sci. 48, 151-280.

Alivisatos, A.P., 1996. Semiconductor clusters, nanocrystals, and quantum dots. Science 217, 933-937.

Alonso, U., Missana, T., Patelli, A., Rigato, V., Ravagnan, J., 2004. $\mu$ PIXE study on colloid heterogeneous retention due to colloid/rock electrostatic interactions. Laboratori Nazionali di Legnaro Annual Report, pp. 68-69.

Berli, C., Piaggio, M., Deiber, J., 2003. Modeling the zeta potential of silica capillaries in relation to the background electrolyte composition. Electrophoresis 24, 1587-1595.

Bradford, S.A., Simunek, J., Bettahar, M., Tadassa, Y.F., van Genuchten, M.Th., Yates, S.R., 2005. Straining of colloids at textural interfaces. Water Resour. Res. 41, W10404 doi:10.1029/2004WR003675.

Brant, J.A., Labille, J., Bottero, J.Y., Wiesner, M.R., 2006. Characterizing the impact of preparation method on fullerene cluster structure and chemistry. Langmuir 22, 3878-3885.

Bruchez, M., Moronne, M., Gin, P., Weiss, S., Alivisatos, A.P., 1998. Semiconductor nanocrystals as fluorescent biological labels. Science 281, 2013-2016.

Chen, K.L., Elimelech, M., 2006. Aggregation and deposition kinetics of fullerene (C-60) nanoparticles. Langmuir 22, 10994-11001.

Derjaguin, B.V., Landau, L.D., 1941. Theory of the stability of strongly charged lyophobic sols and of the adhesion of strongly charged particles in solutions of electrolytes. Acta Physicochim. URSS 14, 733-762.

Duffadar, R.D., Davis, J.M., 2008. Dynamic adhesion behavior of micrometerscale particles flowing over patchy surfaces with nanoscale electrostatic heterogeneity. J. Colloid Interface Sci. 326, 18-27.

Duffadar, R., Kalasin, S., Davis, J.M., Santore, M.M., 2009. The impact of nanoscale chemical features on micron-scale adhesion: crossover from heterogeneity-dominated to mean field behavior. J. Colloid Interface Sci. 337, 396-407.

Filby, A., Plaschke, M., Geckeis, H., Fanghänel, Th., 2008. Interaction of latex colloids with mineral surfaces and Grimsel granodiorite. J. Contam. Hydrol. 102, 273-284.

Hirschey, M.D., Han, Y.J., Stucky, G.D., Butler, A., 2006. Imaging Escherichia coli using functionalized core/shell $\mathrm{CdSe} / \mathrm{CdS}$ quantum dots. J. Biol. Inorg Chem. 11, 663-669.

Jaisi, D.P., Saleh, N.B., Blake, R.E., Elimelech, M., 2008. Transport of singlewalled carbon nanotubes in porous media: filtration mechanisms and reversibility. Environ. Sci. Technol. 42, 8317-8323.

Johnson, P.R., Elimelech, M., 1995. Dynamics of colloid deposition in porous media - blocking based on random sequential adsorption. Langmuir 11, 801-812.

Johnson, P.R., Sun, N., Elimelech, M., 1996. Colloid transport in geochemically heterogeneous porous media: modeling and measurements. Environ. Sci. Technol. 30, 3284-3293.

Kanel, S.R., Nepal, D., Manning, B., Choi, H., 2007. Transport of surface modified iron nanoparticle in porous media and application to arsenic (III) remediation. J. Nanopart. Res. 9, 725-735.

Kloepfer, J.A., Mielke, R.E., Nadeau, J.L., 2005. Uptake of CdSe and CdSe/ZnS quantum dots into bacteria via purine-dependent mechanisms. Appl. Environ. Microbiol. 71 (5), 2548-2557.

Ko, C.-H., Elimelech, M., 2000. The "shadow effect" in colloid transport and deposition dynamics in granular porous media: measurements and mechanisms. Environ. Sci. Technol. 34, 3681-3689.

Kozlova, N., Santore, M.M., 2007. Micrometer scale adhesion on nanometerscale patchy surfaces: adhesion rates, adhesion thresholds, and curvature-based selectivity. Langmuir 23, 4782-4791.

Kuznar, Z.A., Elimelech, M., 2007. Direct microscopic observation of particle deposition in porous media: role of the secondary energy minimum. Colloids Surf. A 294, 156-162.

Lecoanet, H.F., Wiesner, M.R., 2004. Velocity effects on fullerene and oxide nanoparticle deposition in porous media. Environ. Sci. Technol. 38, 4377-4382.

Lecoanet, H.F., Bottero, J.Y., Wiesner, M.R., 2004. Laboratory assessment of the mobility of nanomaterials in porous media. Environ. Sci. Technol. 38, 5164-5169.

Li, M., Ge, Y., Chen, Q., Xu, S., Wang, N., Zhang, X., 2007. Hydrothermal synthesis of highly luminescent CdTe quantum dots by adjusting precursors' concentration and their conjunction with BSA as biological fluorescent probes. Talanta 72, 89-94.

Li, Y., Wang, Y., Pennell, K.D., Abriola, L.M., 2008. Investigation of the transport and deposition of fullerene (C60) nanoparticles in quartz sands under varying flow conditions. Environ. Sci. Technol. 42, 7174-7180.

Luque, A., Marti, A., Nozik, A.J., 2007. Solar cells based on quantum dots: multiple exciton generation and intermediate bands. MRS Bull. 32, 236-241. 
Maynard, A.D., 2006. Nanotechnology: A Research Strategy for Addressing Risk. Woodrow Wilson International Center for Scholars, Washington, DC, p. 45.

Murray, C.B., Norris, D.J., Bawendi, M.G., 1993. Synthesis and characterization of nearly monodisperse $\mathrm{CdE}(\mathrm{E}=$ sulfur, selenium, tellurium) semiconductor nanocrystallites. J. Am. Chem. Soc. 115, 8706-8715.

Pelley, A.J., Tufenkji, N., 2008. Effect of particle size and natural organic matter on the migration of nano- and microscale latex particles in saturated porous media. J. Colloid Interface Sci. 321, 74-83.

Quevedo, I.R., Tufenkji, N., 2009. Influence of solution chemistry on the deposition and detachment kinetics of a CdTe quantum dot examined using a quartz crystal microbalance. Environ. Sci. Technol. 43, 3176-3182.

Ryan, J.N., Elimelech, M., 1996. Colloid mobilization and transport in groundwater. Colloids Surf. A 107, 1-56.

Saleh, N., Sirk, K., Liu, Y.O., Phenrat, T., Dufour, B., Matyjaszewski, K., Tilton, R.D., Lowry, G.V., 2007. Surface modifications enhance nanoiron transport and NAPL targeting in saturated porous media. Environ. Eng. Sci. 24, 45-57.

Scheidegger, A., Borkovec, M., Sticher, H., 1993. Coating of silica sand with goethite: preparation and analytical identification. Geoderma 58, 43-65.

Schroth, M.H., Ahearn, S.J., Selker, J.S., Istok, J.D., 1996. Characterization of Miller-similar silica sands for laboratory hydrologic studies. Soil Sci. Soc. Am. J. 60, 1331-1339.

Simunek, J., van Genuchten, M.Th., Sejna, M., 2005. The HYDRUS-1D software package for simulating the one-dimensional movement of water, heat, and multiple solutes in variably-saturated media - version 3.0, HYDRUS software series 1. Department of Environmental Sciences, University of California Riverside, Riverside, CA. 240 pp.

Song, L., Johnson, P.R., Elimelech, M., 1994. Kinetics of colloid deposition onto heterogeneously charged surfaces in porous media. Environ. Sci. Technol. 28, 1164-1171.
Sposito, G., 2008. The Chemistry of Soils, 2nd ed. Oxford University Press, New York. 330 pp.

Stumm, W., 1992. Chemistry of the Solid-Water Interface. Wiley-Interscience, New York. 221 pp.

Torkzaban, S., Bradford, S.A., van Genuchten, M.Th., Walker, S.L., 2008a. Colloid transport in unsaturated porous media: the role of water content and ionic strength on particle straining. J. Contam. Hydrol. 96, 113-128.

Torkzaban, S., Tazehkand, S.S., Walker, S.L., Bradford, S.A., 2008b. Transport and fate of bacteria in porous media: coupled effects of chemical conditions and pore space geometry. Water Resour. Res. 44 doi:10.1029/ 2007WR006541 (Art. No. W04403)

Torkzaban, S., Hyunjung, N.K., Simunek, J., Bradford, S.A., 2010. Hysteresis of colloid retention and release in saturated porous media during transients in solution chemistry. Environ. Sci. Technol. 44, 1662-1669.

Tufenkji, N., Elimelech, M., 2004. Correlation equation for predicting singlecollector efficiency in physiochemical filtration in saturated porous media. Environ. Sci. Technol. 38, 529-536.

Verwey, E.J.W., Overbeek, J.Th.G., 1948. Theory of the Stability of Lyophobic Colloids. Elsevier, Amsterdam.

Wang, Y., Li, Y., Fortner, J.D., Hughes, J.B., Abriola, L.M., Pennell, K.D., 2008a. Transport and retention of nanoscale C60 aggregates in water-saturated porous media. Environ. Sci. Technol. 42, 3588-3594.

Wang, Y., Li, Y., Pennell, K.D., 2008b. Influence of electrolyte species and concentration on the aggregation and transport of fullerene nanoparticles in quartz sands. Environ. Toxicol. Chem. 27, 1860-1867.

Yao, K.M., Habibian, M.T., Omelia, C.R., 1971. Water and waste water filtration concepts and applications. Environ. Sci. Technol. 5, 1105-1112.

Zhivko, Z., Rumiana, B., Hideki, O., Rajan, J., Yusuke, I., Yoshinobu, B., 2006. Uncoated, broad fluorescent, and size-homogeneous CdSe quantum dots for bioanalyses. Anal. Chem. 78, 321-330. 


\section{DISCLAIMER}

This document was prepared as an account of work sponsored by the United States Government. While this document is believed to contain correct information, neither the United States Government nor any agency thereof, nor The Regents of the University of California, nor any of their employees, makes any warranty, express or implied, or assumes any legal responsibility for the accuracy, completeness, or usefulness of any information, apparatus, product, or process disclosed, or represents that its use would not infringe privately owned rights. Reference herein to any specific commercial product, process, or service by its trade name, trademark, manufacturer, or otherwise, does not necessarily constitute or imply its endorsement, recommendation, or favoring by the United States Government or any agency thereof, or The Regents of the University of California. The views and opinions of authors expressed herein do not necessarily state or reflect those of the United States Government or any agency thereof or The Regents of the University of California.

Ernest Orlando Lawrence Berkeley National Laboratory is an equal opportunity employer. 